\title{
GEOPOLÍTICA Y HOSPITALIDAD: UNA APROXIMACIÓN A LOS ORÍGENES DE LA EXPEDICIÓN DE LOS DIEZ MIL (404-401)
}

\author{
Andrés SÁEz Geoffroy \\ Universidad de la Frontera. Temuco, Chile
}

Resumen: En la discusión sobre la constitución de la expedición de los diez mil generalmente se aducen motivos socioeconómicos. Con el fin de desmitificar lo anterior en el presente trabajo se presenta una aproximación a la influencia que tuvo el nuevo modelo de relaciones exteriores surgido a fines de la guerra del Peloponeso y las relaciones de hospitalidad en la conformación de la expedición de los diez mil.

Palabras clave: Diez mil, Jenofonte, Mercenarismo, Imperio Espartano, Anábasis.

\section{GEOPOLITICS AND HOSPITALITY: AN APPROACH TO THE ORIGINS OF THE EXPEDITION OF TEN THOUSAND (404-401)}

Abstract: In the discussion on the establishment of the ten thousand generally arguesocioeconomicrea sons. To demystify this, in this paper presents an approach to the influence that the new foreign model emerged at the end of the Peloponnesian War and hospitality relationships and their importance in in the constitution of the ten thousand.

Keywords: Ten thousand, Xenophon, Mercenarism, Spartan Empire, Anábasis.

Recibido: 12.03 .13 - Aceptado: 29.04 .13

\section{Correspondencia: ANDRÉs SÁEz G. a.saez01@ufromail.com}

Profesor de Historia Antigua, Historia Medieval y Taller de fuentes clásicas Universidad de la Frontera Temuco, Chile. Magister por Universitat Barcelona (2012) y Profesor de Estado en Historia y Geografía UFRO. Universidad de La Frontera, Temuco, Chile.

Francisco Salazar 1145, Departamento Ciencias Sociales, Facultad de Educación,

Ciencias Sociales y Humanidades. 480869 - 79992898. 


\section{Introducción}

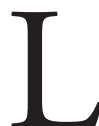

a llamada expedición de los diez mil tiene lugar en una época convulsa para la Hélade. Jenofonte quien escribiera la Anábasis y fuera parte de la expedición como uno de sus líderes, relata y conoce de cerca el fin de la Guerra del Peloponeso y el impacto de esta en la sociedad griega de finales del siglo V. El fin del enfrentamiento significaba el término de la época de oro de Atenas, si bien la muerte de Pericles ${ }^{1}$, la derrota en Mantinea el 418, el desastre en Sicilia, el oro persa y la invasión del Ática anunciaron lentamente la agonía del Imperio Ateniense ${ }^{2}$. El año crucial del 404 Jenofonte rememoraba que los atenienses, una vez enterados de la derrota en Egos Potamos “....Nadie durmió aquella noche, pues no lloraban tan sólo por la suerte de los desaparecidos, sino más bien por su propio destino"3 por último "sitiados los atenienses por mar y por tierra, hallábanse sin saber qué hacer, pues ya no tenían ni naves ni aliados, ni les quedaba trigo....”․ La Guerra había concluido con un triunfo espartano; victoria en realidad a medias, puesto que las últimas campañas espartanas, sobre todo las navales, habían sido financiadas con el oro persa, condición sine qua non para alcanzar la victoria final por parte de los peloponesios ya que estos no poseían el dominio del mar sobre el cual descansaba gran parte del poderío ateniense.

La guerra produjo grandes efectos en la sociedad griega, interesa particularmente en estas ocasión el énfasis en el establecimiento de un nuevo modelo geopolítico de relaciones internacionales a inicios del siglo IV, donde

${ }^{1}$ Para Tucídides, la muerte de Pericles representó un punto de inflexión en la guerra a tal nivel que ninguno de sus sucesores fue capaz de entender su estrategia de guerra ni tampoco poseyeron su genialidad política. (Thu. 2.34).

${ }^{2}$ Kagan señala que la guerra del Peloponeso cambió la faz del mundo griego pero a la vez "suceso trágico, un punto de giro importante en la historia, el final de un período de confianza y esperanza, el comienzo de una época más sombría”. Kagan, D. (2003). Sobre las causas de la guerra y la preservación de la paz. México: FCE. p.28.

${ }^{3}$ X. HG 2.3.1-3.

${ }^{4} \mathrm{X}$. HG 2.3.10. 
factores tales como la geografía, las relaciones de hospitalidad y los ejércitos profesionales cobraron una importancia mayor que en el siglo V, elementos que a su vez coadyuvaron en la constitución por parte de Ciro el joven del ejército mercenario griego más grande conocido hasta ese entonces. De esta manera se sostendrá que los diez mil como cuerpo militar es reflejo de los cambios sociopolíticos del periodo de estudio pero que el énfasis para su constitución se encuentra en la geografía política así como en las relaciones de hospitalidad.

\section{El gran cambio a fines de la guerra del Peloponeso}

En términos generales, la guerra del Peloponeso fue un conflicto que aumentó la escala espacial de los conflictos internos de Grecia, por un lado los límites de la ciudad griega, entendida tanto como territorio como ente estatal fueron traspasados a una lucha de sistemas políticos representados por Esparta o Atenas y sus respectivos aliados; mientras desde lo geográfico el conflicto se extendió todo el mundo griego conocido. Esta lucha sin cuartel, dejó desgastadas las estructuras políticas griegas, lo que permitiría que Macedonia a la postre se alzara como potencia ${ }^{5}$. Así mismo estallaron una serie de problemas sociales ya que la ciudadanía dejaba de garantizar algún bienestar material debido a la crisis económica y social subsiguiente ${ }^{6}$.

Para la vencedora Esparta, el desgaste provenía en cuanto la muerte del Homoioi implicaba un vacío que no podía llenarse, salvo con un hijo de otro Homoioi y recién cuando este tuviera 19 años, "Esta escasez de hombres en la que los antiguos han visto y con razón el infortunio mortal de Esparta, resulta en primer lugar de la guerra, que casi endémica, diezma la población masculina en edad de servir"7. La situación en cierto sentido era crítica por cuanto todo el aparato militar se orientaba al control interno (por el sistema de la $\alpha \gamma o \gamma \varepsilon$ y el dominio de los ilotas), a lo que se sumaba el peso incipiente del control de colonias ${ }^{8}$, lo que requirió

${ }^{5}$ Cfr. Hanson V. "Epaminondas el tébano y la doctrina de la guerra preventiva” en Hanson V., ed. (2012) El arte de la guerra en el mundo antiguo: de las guerras persas a la caida de Roma. Barcelona: Crítica.

${ }^{6}$ Respecto a la crisis ver Rouzé F. y Amouretti M. (1992) El mundo griego antiguo, España: Akal. pp.197-199; así mismo la República de Platón o sus Leyes intentan ser soluciones ideales a los modelos políticos de la época a modo de reconvención por los problemas existentes.

${ }^{7}$ Jardé. A (1960).La formación del pueblo griego, México: UTEHA. p.116-117.

${ }^{8}$ De acuerdo a Parke el Imperio espartano tenía tres objetivos a cumplir 1) garantizar la lealtad de las ciudades conquistadas y de las oligarquías locales junto con la amenaza interna de los ilotas 2) la administración de territorios bajo el sistema de decarquías y harmostes, estos últimos con poder sobre tropas y amplios territorios y 3) la percepción tributaria con la cual se contrataban 
un contingente mayor de hombres. De esta manera los tributos percibidos del control territorial del llamado Imperio espartano se orientaron en muchos casos a la contratación de ejércitos mercenarios?

Por su parte, la derrotada Atenas, pasaba de una oligarquía instaurada por Esparta nuevamente a la democracia mediante un golpe de mano de Trasíbulo el 403, aceptando los espartanos la situación para mantener el status quo. En todo caso esta restauración distaba bastante de devolver a la polis su antiguo poderío naval y geopolítico el cual se renovaría en parte con apoyo persa al fundarse la llamada segunda liga délica el 377. Irremediablemente las condiciones de paz impuestas a Atenas, así como la vigilancia que Esparta tuvo que acometer respecto a sus aliados con el fin de evitar que renaciera un conflicto bélico, trasladó el foco de las acciones políticas de manera coyuntural al Asia menor.

Bajo la lógica del conflicto, la guerra evolucionó de lo estrictamente táctico a una dimensión ante todo estratégica debido a la amplitud en las escalas de los enfrentamientos bélicos tanto en un plano temporal como espacial, para Austin y Naquet, entre 431 y 338 hubo un estado de guerra constante, tanto que "Si algo caracteriza al siglo IV es la guerra" ${ }^{10}$. Es relevante en este aspecto la cuestión del tiempo ya que mientras una o dos batallas definían la suerte del conflicto (como el caso de las guerras médicas) un asedio en cambio requería tiempo y soldados permanentes, no campesinos que tuvieran que retirarse en época de cosecha ${ }^{11}$. El inicio de los asedios como técnica militar de larga duración, unido a la muerte de los ciudadanos provocó que hubiera echar mano a soldados especializados de tiempo completo: los mercenarios, sean estos de infantería pesada o peltastas ${ }^{12}$. Como señala Sage es raíz de estos antecedentes que se deriva la creación de una clase militar no ciudadana en carácter de fuerzas armadas permanentes y cuya única función fuese lo militar, esto se tradujo en que a fines de la guerra del Peloponeso el uso de mercenarios se había generalizado ${ }^{13}$.

diferentes tropas entre ellas mercenarias. Parke H. W . (1930) "The Development of these condspartan Empire (405-371)", The Journal of Hellenic Studies, 50/1, 37-79.

${ }^{9}$ Marinovic L. (1998) Le mercenariatgrecau 4e siècleavantnotreère et la crise de la polis. Paris: Belles Lettres. P.22.

${ }^{10}$ Austin M y Vidal-Naquet P. (1986) Economía y sociedad en la antigua Grecia. Barcelona: Paidos. p.130.

${ }^{11}$ El caso espartano es más claro aún, no podían abandonar el Peloponeso so pena de una rebelión de ilotas o que los aliados cambiaran de bando e iniciaron nuevos conflictos, lo anterior orientó la política militar espartana a la seguridad interna.

${ }^{12}$ Austin M y Vidal-Naquet P Op.Cit. p.131.

${ }^{13}$ Sage M. (2003). Warfare in ancient Greece: a sourcebook. Londres: Routledge. p. 147. 
En general la guerra del Peloponeso había provocado un claro desgaste interno de las poleis además de producir una manifiesta alteración el juego político dentro del Imperio persa, los sátrapas de Asia menor no habían facilitado oro, barcos y tripulaciones a los espartanos de manera gratuita ni tampoco por mero afán caritativo. El apoyo más relevante había provenido de Ciro el joven, karanos (señor) de las llamadas satrapías marítimas (Jonia) y uno de los hijos del fallecido rey Dario II. Ciro, hermano por tanto, del recién asumido rey Artajerjes II (404) había dado su apoyo a Esparta esperando una devolución del esfuerzo aportado a la victoria de los peloponesios ${ }^{14}$. Aprovechando la coyuntura del fin de la guerra en la Hélade se asoció a una serie de agentes de reclutamiento cuyo fin era enganchar soldados para una expedición al interior contra su hermano para apoderarse de esta manera del trono, esperaba también que se le uniera un cuerpo peloponesio por la alianza sellada con Esparta. El cuerpo griego bajo su servicio sería conocido como los diez mil constituyéndose en el primer cuerpo militar a gran escala que jugaba en el espacio griego un papel particular y relevante.

Para Trundle los diez mil son la primera experiencia militar de tipo mercenario a gran escala, si bien se debe señalar que este fenómeno no respondería únicamente a fines económicos y de obtención de riqueza por parte de los mercenarios, sino que también influiría el grado de amistad, $\xi \varepsilon v o r$, existente entre los participantes de la expedición ${ }^{15}$. El mercenarismo, como señala Duran Badell, es un fenómeno histórico común en el mundo griego y para nada novedoso hacia el siglo IV, existen referencias que hacen posible datar mercenarios griegos en el siglo VII en Egipto y Medio oriente ${ }^{16}$. El fenómeno en todo caso hacia el siglo V se transformaría en cuanto magnitud con la expedición de los diez mil, ya que las condiciones históricas de la Hélade habían variado una enormidad a partir del fin de la guerra del Peloponeso ya que el ejército ciudadano del siglo V, había sido reemplazado por un ejército contratado y conformado por soldados pagados.

Algunos historiadores, en general de la décadas de los 70 y 80 del siglo XX, han centrado el fenómeno mercenario de los diez mil en otro tipo de apreciaciones, sobre todo de índole socioeconómica ${ }^{17}$. En general sostienen que con el

${ }^{14} \mathrm{X} . \mathrm{HG} 3.1 .1$.

${ }^{15}$ El concepto ( $\left.\xi \varepsilon v o 1, \xi \varepsilon v o \sigma\right)$ que significa extranjero o amigo extranjero, se usa de manera general desde época clásica para designar los mercenarios. Trundle M (2004). Greek Mercenaries From the Late Archaic Period to Alexander. Inglaterra: Routledge. p.45, p.91 Cfr. Jenofonte y su Anábasis. Donde el autor griego también la utiliza como sinónimos. El término se usa como aliado.

${ }^{16}$ Duran Badell M. (1998) "El mercenario en la Grecia antigua", Militaria. Revista de cultura militar, 12, 89-101, pp.90-91. 
empeoramiento de las condiciones sociales de la Hélade a fines del siglo V se asiste a la expulsión de una masa empobrecida de las poleis, la que encontraría en el mercenarismo una forma de buscar riqueza y por medios pacíficos o violentos la ciudadanía perdida ${ }^{18}$. Al respecto la obra de Nussbaum es paradigmática de este tipo de explicaciones, ya que sostiene que los diez mil son un ejército helénico donde el factor geográfico no contaba para nada, transformándose así en una comunidad política constituida por tres niveles de participación, el de los soldados comunes equivalentes a los ciudadanos, los capitanes quien serían la conexión con las capas dirigentes y los generales quienes se habrían transformando en la clase dirigente y de gobierno de la comunidad política de los diez mil similar a una polis perfecta ${ }^{19}$.

En este caso, juzgamos necesario enmarcar esta expedición dentro de un concepto político particular, bajo este prisma Thomson, señala que el mercenario es un individuo que ejerce violencia en el sistema internacional, y que al no depender de un Estado en particular, puede ser usado para participar en el juego de las políticas exteriores por parte de cualquier actor dentro de dicho panorama ${ }^{20}$. La violencia es usada por una determinada causa, en este caso la razón de Estado de ejercer violencia sobre un objetivo en particular por lo que al mercenario se le paga no tanto para sí como para un cumplir un claro objetivo político, no existiendo grandes diferencias en este sentido con otras profesiones en cuanto a la realización de algún oficio.

El historiador Daniel Gómez Castro, ha centrado aún más el debate acotándolo y retomando el mercenarismo del siglo V y IV desde los planos de la política exterior, "En el terreno de la política, los mercenarios contribuyeron al desarrollo de unas necesarias relaciones internacionales, y en el terreno bélico al afianzamiento de nuevas formas de hacer la guerra en Grecia que se ajustaban mejor a la situación política generada por los mencionados pactos internacionales" 21 . Es así que para Gómez Castro se debe rechazar la idea del mercenarismo en el mundo antiguo como algo residual de las condiciones socioeconómicas, del mundo griego del siglo IV, apreciaciones que se corresponderían con nuestra visión

${ }^{17}$ Cfr. Duran Badell. Op.cit.

${ }^{18}$ Rouzé y Amouretti. Op. Cit. p. 167.

${ }^{19}$ Cfr. Nussbaum G.B. (1967). The Ten Thousand: A Study in Social Organization and Action in Xenophon'sAnabasis. Leiden, Holanda: EJ Bill.

${ }^{20}$ Thompson. J. (1990) "StatePractice, International Norms, and the Decline of Mercenarim", International Studies Quarterly 34, 23-47, p.34-35.

21 Gómez Castro D. (2011) "La Campaña egipcio-chipriota (383-373 a.C.): relaciones 
sobre los mercenarios actuales ${ }^{22}$. Para el citado autor ya, hacia inicios del siglo IV se había producido un cambio fundamental en las relaciones internacionales del Mediterráneo oriental ${ }^{23}$. Las graves pérdidas a todo nivel producidas por la guerra del Peloponeso llevó a que los Estados transformarán los mercenarios en instrumento fundamental de la realización de su política exterior; por tanto más allá de la situación socio económica, es el nuevo modelo internacional -a juicio de Gómez Castro y nuestro- el que impulsa la existencia de ejércitos mercenarios a gran escala, el primero de ellos el de los diez mil, si bien el autor hace notar que entre el periodo de la expedición de Ciro hasta el 373 son más de 20.000 los mercenarios que fueron enrolados por los persas ${ }^{24}$.

Veamos ahora el tema de su procedencia, la geopolítica y los lazos de hospitalidad en la creación de este cuerpo mercenario y como estos elementos interrelacionados dieron origen a este primer cuerpo mercenario a gran escala.

\section{Geografía y geopolítica en la expedición de los diez mil.}

En palabras del mismo Jenofonte los arcadios y los aqueos representaban la mitad de los alrededor 13.000 mercenarios enrolados. Sin duda es imposible a pesar del dato aportado por Jenofonte identificar la procedencia de todos y cada uno de los soldados ${ }^{25}$. El ateniense menciona a 65 personas entre generales, capitanes y soldados de los cuales es posible realizar un seguimiento de apenas un puñado de ellos ${ }^{26}$. En general es posible señalar que de los 65, un tercio proviene del eje geográfico Arcadia-Acaya seguido por participantes de Atenas y Esparta, si bien debemos comprender estas cifras dentro de un margen acotado en la relevancia que Jenofonte otorga a cada participante. De los restantes podemos encontrar mercenarios de Acarnania, Acaya, Ambracia, Arcadia, Argos, Beocia, Creta, Dardania, Elea, Mégara, Quios, Sicilia, Tebas, Tracia y Tesalia entre otras procedencias. Dentro de ese marco numérico, se debe acotar aún más a las elites dirigentes de la expedición de los diez mil, es posible señalar que se repiten en los mandos de la expedición el mismo patrón respecto que la tropa en general, un tercio de los generales procede de Arcadia y un cuarto del total son aqueos ${ }^{27}$. La

internacionales y mercenarios griegos en oriente"- Gladius, 31, 43-56. p. 52.

22 Gómez Castro D. (2010) "El mercenarios en el mundo griego a la luz de los estudios contemporáneos: reflexión teórica y nuevas tesis” Habis, 41, 95-115. p.96.

${ }^{23}$ Gómez Castro, D. (2011) "La campaña egipcio-chipriota..." Op. cit. pp. 52-53.

${ }^{24}$ Ibíd. p. 52.

${ }^{25}$ X. An. 6.2.9-11.

${ }^{26}$ Marinovic L. Op.cit pp.33-34, también Radin M. (1911) "Xenophon's Ten Thousand” The Classical Journal, 7, 51-60. 
excepción en todo caso se produce en que dentro de dichas cifras generales los atenienses y espartanos acumularon más poder que los arcadios y aqueos, por lo que se puede inferir una clara diferenciación entre lo cuantitativo y lo cualitativo.

Por lo anteriormente señalado, los números no expresan sino un mero formalismo ya que dentro de la cadena de mando se vislumbra una expresión concreta de las relaciones geopolíticas de la Hélade. Son los mismos arcadios y aqueos los que enuncian una discusión que sin duda debe haber sido constante -y hasta lógica- de que si bien más de la mitad de los mercenarios eran arcadios o aqueos,se encontraban bajo el mando de un espartano (Clearco o Quirísofo) o bien de un ateniense (Jenofonte). Jenofonte enuncia lo anterior por boca de Calímaco de Parrasia y Licón de Arcadia, a través de un discurso en que declaran que era vergonzoso para los aqueos y arcadios que hubieran generales, en clara referencia a Jenofonte, que no habiendo aportado tropas a la expedición optaran a mandar ${ }^{28}$. Lo anterior desde mi perspectiva es demostrativo de lo que fue el modelo de las relaciones geopolíticas de Grecia posterior a la guerra del Peloponeso, siendo un reflejo de las nuevas formas del juego geopolítico en el espacio histórico Egeo. La afirmación anterior tiene su base en que desde el punto de vista de la geografía histórica, así como de la arqueología es posible establecer tres tipos de regiones históricas para Grecia en los siglos V y IV ${ }^{29}$.

Las primeras regiones denominadas por la geografía histórica como 'heartland' ${ }^{30}$ por su peso político pero también demográfico se transformaron en los grandes centros históricos del siglo V. Tanto Atenas, como Beocia y Esparta fueron aquellas polis que a finales del siglo IV por las condiciones antedichas pueden ser consideradas como 'Heartland' dentro del mundo griego antiguo $^{31}$. Una segunda zona de las regiones es el llamado 'hinterland', es decir aquellas regiones donde las potencias del 'heartland' desarrollan todo su poderío e influencia de manera directa y que para el caso griego se correspondería con las regiones de Arcadia, Argólida, Acaya y Tesalia, menos desarrolladas que las anteriores en cuanto sistema político y urbanismo ${ }^{32}$. Por último se encuentran

${ }_{27}^{27}$ Marinovic, L. Op.cit pp.33-34.

${ }^{28}$ X. An. 6.2.9-11.

${ }^{29}$ Cfr. Binttliff, J. (1997) "Regional Survey, Demography, and the Rise of Complex Societies in the Ancient Aegean: Core-Periphery, Neo-Malthusian, and Other Interpretive Models" Journal of Field Archaeology, 24, pp. 1-38.

${ }^{30}$ El heartland se refiere a los núcleos de donde emana poder en todos los sentidos y con énfasis en la expansión. Los conceptos de hinterland y heartland son de uso común en la geopolítica y no tienen una traducción conceptual equivalente.

${ }^{31}$ Binttliff, J. Op.cit. pp. 20-22. 
las regiones periféricas siendo aquellas las que los mismos griegos denominaron como bárbaras tales como el Epiro y Macedonia ${ }^{33}$. Bajo este análisis y de acuerdo a lo anterior, Jenofonte en su Anábasis lo que hace es presentar el nuevo esquema de poder de las relaciones exteriores.

Para el caso de Esparta, Jenofonte a través de Clearco y Quirísofo muestra el superior y victorioso poder de Esparta en la guerra del Peloponeso, su pro espartanismo es inclusive una muestra de su realismo político, El mando del ejército debe ser ostentado por un lacedemonio ya que ellos son los vencedores de la guerra del Peloponeso ${ }^{34}$, el mismo Jenofonte rechaza el mando cuando se le ofrece porque "si yo, que he visto estos actos, decidiera invalidar su dignidad [la de los espartanos] allí en donde pudiera, tengo la sospecha de que muy pronto sería castigado por ello." 35 Tanto así es que por medio de un dialogo, en boca de Quirísofo lanza una mordaz crítica a la democracia ateniense ${ }^{36}$.

Si bien Atenas se encontraba acabada el 404, en la época que Jenofonte escribe (hacia 370) gracias al oro persa había recuperado algo de su esplendor, al menos había logrado crear una nueva liga délica y ser parte del concierto internacional debido a una rápida recuperación política y económica ${ }^{37}$. Jenofonte a través de su propio personaje demuestra a esa Atenas con algo de poder en el concierto político o al menos, como potencia histórica. Lo hace siempre por medio de discusiones en boca de Ciro al alabar la libertad de los griegos ${ }^{38}$, emulando al recurso adoptado por Heródoto para explicar la victoria sobre los persas en las guerras médicas en cuanto la libertad es un valor superior al de la esclavitud, esta última netamente persa ${ }^{39}$. Por último los beocios son parte importante del contingente demostrando su alza en cuanto su poderío político y militar. El mismo Jenofonte se transforma en participante de la expedición por instigación de Próxeno de Beocia y no de mottu propio ni por conocer personalmente a Ciro $^{40}$. En tanto otros generales, salvo los tésalos por lo bueno de Arístipo y lo malo de Menón ${ }^{41}$, no ocupan gran parte del análisis de Jenofonte.

\footnotetext{
${ }^{32}$ Ibíd. pp.20-22.

${ }^{33}$ Baste recordar la respuesta dada a Alejandro de Macedonia de por qué no podía participar en los juegos olímpicos, Hdt. 5.22.

${ }^{34}$ X. An 6.1.27-28.

${ }^{35} \mathrm{X}$. An6.1.27.

${ }^{36}$ X. An4.6.16.

${ }^{37}$ Baron, C. (2006) "The Aristoteles Decree and the Expansion of the Second Athenian League" Hesperia: The Journal of the American School of Classical Studies at Athens, 75, 379-395.

${ }^{38}$ X. An1.7.3.

${ }^{39}$ Hdt. 6. 112.

${ }^{40}$ X. An3. 1.14 .
} 
De esta manera son los tres centros de poder (con especial énfasis en Esparta), los que poseen el derecho y deber del mando, algo que se encuentra en la misma dirección de la descripción que nos da Jenofonte respecto de Clearco como disciplinado y que sabe resolver situaciones conflictivas ${ }^{42}$. A su vez la gran masa de soldados anónimosocupan un lugar marginal dentro del reflejo global de este juego político como si fueran una clientela militar. Los hechos anteriores ayudan a esclarecer cómo y por qué se conformaron los diez mil. Más allá de causas de tipo socio-económico, desde nuestra perspectiva sería la $\xi \varepsilon v i \alpha y$ las relaciones de hospitalidad, ancladas a una dimensión geográfica,las que explicarían de mejor manera las bases sobre la cual se conformó el primer ejército mercenario a gran escala. ${ }^{43}$ De esta forma, las relaciones y conexiones sociales se transforman en un reflejo del juego geopolítico y se plasman en una expedición útil para los griegos, en particular para Esparta pues es capaz de debilitar al Imperio persa, conocer sus defensas y grado de reacción en términos militares.

\section{Sobre la $\xi \varepsilon v 1 \alpha$ y las relaciones de amistad en la Anábasis.}

Del análisis de la procedencia realizado anteriormente, se puede postular que el peso geográfico y las relaciones sociales ancladas al territorio tuvieron una influencia relevante en la conformación de la expedición de los diez mil. A nuestro juicio es poco el interésque ha tenido el análisis de las relaciones hospitalidad y amistad en el origen de los diez mil, razones que podrían tener un mayor peso que la generalización de tipo económico. De esta misma manera está atestiguado que de las poleis derrotadas en clara referencia a Atenas, la recuperación económica avanzó a un ritmo sorprendente ${ }^{44}$, así mismo Esparta con el control de su Imperio tampoco tuvo serios problemas económicos. De esta forma se hace necesario inferir las motivaciones sobre las cuales se enrolaron los diez mil e ir más allá del economicismo y centrarnos en las relaciones de amistad y hospitalidad. Al respecto, es necesario aclarar que los mercenarios no recibían una paga superior a los ejércitos ciudadanos y su enrolamiento tampoco es señal de que mejoraría la situación de los grupos sociales pauperizados. Como apunta Gómez Castro hay mercenarios porque hay una demanda de ellos y quienes le

${ }^{41}$ X. An2. 6.21.

${ }^{42}$ X. An2. 6.1-15.

${ }^{43}$ Trundle M (2004). Greek Mercenaries From the Late Archaic Periodto Alexander. Inglaterra: Routledge. p. 45.

${ }^{44}$ Forni C. y Plácido D. (2009) "De la guerra del Peloponeso a la paz del Rey (III): los factores 
contratan no son generales privados sino que son Estados en su forma de poleis, Imperio Persa, monarcas o bien satrapías, etc ${ }^{45}$.

Debido a lo anterior, podemos afirmar que la $\xi \varepsilon v i \alpha$ significó un proceso básico sobre el cual se asentó la construcción de la expedición de los diez mil. La empresa se constituye sobre los deseos de Ciro de convertirse en rey persa, pero más allá de sus motivaciones personales, Ciro intuye desde un principio que el apoyo a algún bando en la guerra del Peloponeso repercutiría favorablemente en su situación estratégica sobre todo si se trataba del ganador. Bajo este prisma su apuesta por apoyar con todos sus recursos disponibles a los espartanos es una jugada política sobre la cual edifica un ejército, teniendo un cuerpo hoplítico de gran número y superior a las tropas persas normales ${ }^{46}$. La otra apuesta política existente de debilitar a espartanos y atenienses por igual deseada por Farnabazo es descartada por Ciro el que seguramente planeaba por entonces su futura expedición ${ }^{47}$.

No es arbitrario que se haya decantado por Esparta; disponía de las mejores redes de reclutamiento de hoplitas, junto con la capacidad de movilización marítima hacia Jonia, más que mal una potencia marítima como Atenas no servía para ayudar en un avance interior hacia Media como lo era el objetivo de Ciro, los barcos como se demostraríano fueron de gran de utilidad en estas expedición.El financiamiento persa de la victoria Espartana sirvió para que Ciro les recordara a los lacedemoniosdicha amistad o alianza, con el fin de convocarlos a la expedición del interior, los éforos vieron en esta petición algo lógico y para nada descabelladoindependiente del objetivo que les haya comunicado a los generales ${ }^{48}$. Mediante este mecanismo reclutó una serie de líderes por las diferentes relaciones de hospitalidad construidas ${ }^{49}$; estos generales son los que iniciaron la campaña de reclutamiento mercenario.

De acuerdo a lo anterior, el general más relevante de acuerdo Jenofonte es Clearco de Esparta, quien había sido harmosta de Bizancio, y al parecer exiliado por los éforos. Se transformó en el más importante para Ciro no tanto por el

económicos públicos y privados en Atenas”. Gerión, 27, 147-160. pp.148-150.

${ }^{45}$ Gómez Castro D. (2010) “El mercenario en el mundo griego...” Op. cit. p. 101.

${ }^{46} \mathrm{X}$. HG 1.5.3.

${ }^{47}$ X. HG 1.5.3, además Sage. Op.Cit p.148 señala que la demanda persa fue siempre a favor de reclutar infantería pesada, puesto que el ejército persa carecía de ella. Ver también respecto al armamento pesado Whitehead D. (1991) "Who Equipped Mercenary Troops in Classical Greece?” Historia: Zeitschriftfür Alte Geschichte, 40,105-113.

${ }^{48}$ X. HG 3.1.1-2. 
número de su tropa como por las cualidades al mando de las mismas así como por ostentar el mando de gran parte del ejército griego de Ciro. Las causas de por qué fue exiliado no están del todo claras, puesto que las versiones difieren entre sí, para Jenofonte se trataría de un exiliado por haber perdido Bizancio a manos de los atenienses como lo relata en sus helénicas ${ }^{50}$. Diodoro Sículo en cambio declara que fue al exilio por comportarse de manera tiránica tanto que se enfrenta a un ejército espartano que venía a alejarle del mando ${ }^{51}$, pero, a pesar de aquello se alza con el mando de todos los lacedemonios ${ }^{52}$. Plutarco -el que seguramente tuvo acceso a otras fuentes- señala que los espartanos le dieron la orden de ponerse bajo Ciro enviándole la correa, esto es un mensaje secreto quizá con miras a rehabilitarle ${ }^{53}$.

La versión del destierro de Clearco, defendida por Jenofonte podría estar en concordancia con el hecho de que de ser desterrado de Esparta, su actuación no obligaba en ningún grado a Esparta a asumir los costes políticos de la presencia de Clearco al mando de los diez mil, beneficiándose Esparta tanto en el caso de la derrota de Ciro como de su victoria ${ }^{54}$, tal como se demostró posteriormente con la expedición de Agesilao el 399 en Asia menor. Más que mal los lazos de $\xi \varepsilon v i \alpha$ obligaban a Esparta a enviar la ayuda prometida sin importar la forma, lo importante era cumplir el pacto y los mercenarios eran una buena forma de cumplir lo firmado y eludir una futura responsabilidad.

Retomando lo anterior, todas las versiones coinciden en que Clearco entró en tratos con Ciro en su época de actuación en el Quersoneso y que Ciro encantado con la personalidad del general le habría solicitado su apoyo convirtiéndolo en su huésped y aliado ${ }^{55}$. Así mismo Clearco, siendo harmosta tenía a su disposición tropas lacedemonias y megarenses como se menciona en la helénica ${ }^{56}$; hay que hacer notar que en la expedición de los diez mil se encuentra un destacamento megarense de 300 hombres al mando de Pasión de Mégara, que Jenofonte no aclara como llegaron por lo que esta podría ser la forma de como esos 300 megarenses se unieron a la expedición, es decir las relaciones de hospitalidad podrían explicarlos movimientos de tropas de un lugar a otro según lo requiriese el panorama geopolítico.

\footnotetext{
${ }^{49}$ X. An 1.1.6; Así mismo Jenofonte usa $\xi$ cvl $\alpha$ como sinónima de ambas.

${ }^{50}$ X. HG 1.3. 14-22.

${ }^{51}$ D. $S$ 14.16.1-5.

52 D. S 14.19 .8$.

${ }_{53}^{5}$ Plu. Art. 6.

${ }^{54}$ Justino 5.11.

${ }^{55}$ X. An2.6.1.
} 
Dentro de esta misma argumentación Jenofonte se unió a la expedición por los lazos de amistad que tenía con Próxeno de Beocia ${ }^{57}$. El fin como confiesa era obtener la amistad de Ciro seguramente con miras a futuras acciones políticas, a su vez el mismo Próxeno buscaba alcanzar riqueza y renombre de la amistad de $\mathrm{Ciro}^{58}$. En todo caso esta $\xi \varepsilon v i \alpha$ a mi juicio no es posible circunscribirla netamente a los altos mandos. Un ejemplo notorio se produjo en el hecho de que una vez capturados y asesinados Clearco, Próxeno, Menón, Sócrates de Acaya y Arístipo de Tesalia, el mando recayera en generales que son coterráneos o bien asistentes de los generales muertos (por ejemplo Timasión de Dardania que reemplaza a Clearco). Por tanto podría señalarse al respecto que la $\xi \varepsilon v i \alpha$ actuaba a nivel interior en las relaciones entre el líder y los mercenarios reclutados a modo de clientela militar, esto mismo se observa con los generales arcadios y aqueos, muertos los generales son reemplazados por gente de la misma tierra.

Es con los aqueos y arcadios donde las relaciones exteriores y la $\xi \varepsilon v i \alpha$ cobraron una mayor importante. A Jenias de Arcadia quien poseía una relación de hospitalidad con Ciro, se le había otorgado el mando de todas las guarniciones de Jonia ${ }^{59}$, así mismo se señala que Ciro invoca a Sofeneto de Estinfalia, y Sócrates de Acaya basándose en los lazos de amistad ${ }^{60}$. Desde ese punto de vista, que la gran cantidad de mercenarios hayan sido de Acaya o Arcadia tiene una serie de explicaciones plausibles. Por ejemplo el requisito de que los soldados fueran peloponenses $^{61}$, impulsó que la paz en la Hélade lograda por los espartanos alejara los conflictos y los lacedemonios utilizando sus relaciones diplomáticas exhortaran a aqueos y arcadios para a ir Jonia mediante relaciones de amistad, ya que estos habían actuado como mercenarios durante la guerra del Peloponeso, así se evitaba una posible rebelión en el Peloponeso ${ }^{62}$. Dentro de lo anterior los espartanos tenían la costumbre de enviar a otros peloponesios a cumplir sus alianzas o bien a socorrer ciudades como lo había sido el caso de Etolia el 427, donde "enviaron tres mil hombres de sus aliados, todos muy bien armados" 63 , y que no era muy distinto de lo que ocurrió con Ciro.

En segundo lugar estas regiones existía un incipiente potencial demográfico al no verse involucradas directamente en las guerras, Acayase encontraba en

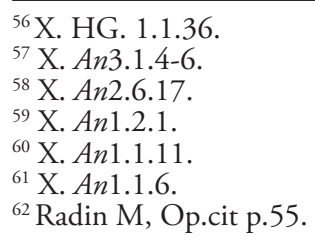


pleno despegue demográfico, mientras en Arcadia se vivía el clímax de un proceso similar, Bintliff agrega que el desarrollo de este proceso fue explosivo para ambas regiones ${ }^{64}$. A raíz de lo anterior podría señalarse que existían recursos humanos para ser contratados como mercenarios, sobre todo en estas regiones tradicionalmente abastecedoras de soldados, al contrario de otras tierras más afectadas por la guerra. A su vez la sociedad arcadia era tribal, el mismo Estrabón ${ }^{65}$, que escribiera quinientos años después, en su geografía hace mención a aquello, esto aumentaría las probabilidades de reclutamiento puesto que podrían haberse enlistado tribus completas mediante sus lazos familiares en relación a un general con el cual tuvieran lazos de hospitalidad. Esto podría haber facilitado el reclutamiento de aquellos grupos donde el crecimiento demográfico habría agotado los recursos naturales o bien en las áreas montañosas donde el hábitat no alcanzara a mantener la población. Esta $\xi \varepsilon v i \alpha$ logró involucrar líderes que facilitaron el reclutamiento de tropas activas y mercenarias, ya queen esto se basaban las relaciones que Ciro estableció con los generales de manera personal.

Otro tipo de $\xi \varepsilon v 1 \alpha$ que podemos observar es la de Arísitpo de Tesalia. Los tésalos en particular la dinastía alévada ${ }^{66}$ de la cual formaba parte el citado Aristipo, eran aquellos tésalos que en la época de las guerras Médicas se había puesto de parte de los persas ${ }^{67}$ generándose una lucha de partidos por el control de Tesalia, por lo que las relaciones de amistad y hospitalidad pueden ser retrotraídas inclusive a ámbitos familiares en conjunto con los exilios de tipo político; era lo que había sucedido con Temístocles, Hipias y Cleombroto sin ir más lejos ${ }^{68}$. En este caso se transformaba en parte fundamental de las relaciones políticas del Egeo, un sujeto al ser un exiliado y aceptar el apoyo al rey persa se transformaba en un huésped de este bajo el cual al aceptado se le daban colonias, ciudades, tierras o inclusive el mando de tropas como sucedió con Arístipo de Tesalia para apoyarlo en su lucha de partidos y disponer de un gobierno aliado en la Hélade.

Se observa también otra forma de alcanzar el mando, en conexión con las relaciones afectivas o sociales, como muy bien apunta Hindley en la Anábasis

\footnotetext{
${ }^{63}$ Tuc 3.95.

${ }^{64}$ Bintliff. Op.cit. pp. 20-22.

${ }^{65}$ Strabo. 8.8.1.

${ }^{66}$ Hdt. 7.132.

${ }^{67}$ Hdt.7.172-173. Además sobre el exilio ver Westlake H. D., (1936) "The Medism of Thessaly," The Journal of Hellenic Studies, 53, 12-24.

${ }^{68}$ Temistocles Th.1.15, Pausanias Th.1.4, Temistocles Plut.Them. 31, Hipias Th 6.59.3, Demarato Hdt 6.70, Cleomenes Hdt 6.74.
} 
podemos encontrar diversas expresiones de afectividad ${ }^{69}$. El mismo Arístipo de Tesalia supuestamente por un amorío con Menón le había otorgado a este siendo muy joven el mando de tropas. El mismo Menón, sindicado como el que traicionase a los generales griegos se habría salvado por tener una $\xi_{\varepsilon v i \alpha}$ de carácter amoroso con Arieo, persa cercano a Ciro pero que una vez muerto se transformó en aliado de Artajerjes. No obstante no es posible determinar si esto relativo a la intimidad de Menón es cierta o constituye una estrategia para desacreditarlo. Si bien la relación de tipo pederastica eran cuestiones comunes ${ }^{70}$ que podrían haber servido para fomentar los grados de hospitalidad y amistad.

Retomando el tópico de la captación de los diez mil por causa económicas es imposible negar la necesidad de obtener riqueza ${ }^{71}$. Ahora bien esto se presenta de variadas formas dentro de la Anábasis. La riqueza por sí sola no es garantía particular de nada, refiriéndose a los capitanes, aquellos conectores ${ }^{72}$ que de alguna manera ya conocían la guerra de seguro, Jenofonte seńala que antes de la guerra tenían más riquezas y prestigio ${ }^{73}$. Para otros esta misma riqueza se asocia no a una necesidad material sino a recuperar y mantener un determinado status perdido en la ciudad de origen con la instalación de las oligarquías y las constantes guerras civiles, las que habían generado un movimiento de personas bastante amplio en toda Grecia producto de los emigrados que expulsados requerían trabajo en un lugar donde no importase su origen ni su causa de llegada (mercenario), es el caso por ejemplo de Dracontio de Esparta, exiliado por el asesinato de un espartiata ${ }^{74}$.

Los cambios políticos provocaron la pérdida de los derechos de ciudadanía, los que podrían ser recuperados mediante la riqueza para tener un status. El mismo Arístipo de Tesalia se encontraba con Ciro a favor debido a la lucha de partidos sostenida en Tesalia, junto a él se encontraría Menón. En tanto Timasión de Dardadania era otro caso similar; desterrado, esperaba ser recibido en su patria por las riquezas conseguidas asociándose así la idea de riqueza y recuperación

${ }^{69}$ Cfr. Hindley C. (1994) "Eros and Military Command in Xenophon” The Classical Quarterly, New Series, 44, 347-366.

${ }^{70}$ Aproximadamente Menón menos de 25 y Arístipo sobre los 35. Ver en particular Hindley C. Op.Cit p.20.

${ }^{71}$ Miller H. (1984) “The Practical and Economic Background to the Greek Mercenary Explosion” Greece \& Rome, Second Series, 31,153-160. Y. Russel A.G. (1942) "The Greek as a Mercenary Soldier" Greece \& Rome, 11/33, 103-112.

${ }^{72}$ Nussbaum. Op. Cit. pp.36-39.

${ }^{73}$ X. An3.1.37.

${ }^{74}$ X. An4.7.25. 
de status ${ }^{75}$. El caso contrario lo demuestra Ceratadas de Tebas, pro espartano que buscaba el mando de tropas pero cuya salida de Tebas no se debía al exilio ¿Qué habrá querido decir Jenofonte cuando menciona que no había salido por exilio? ${ }^{76}$, dicha distinción podría significar que la mayoría de los participantes eran exiliados o bien se encontraban de manera obligada presentando el servicio de armas, una "obligación" que podría haber provenido tanto de la $\xi \varepsilon v i \alpha$ con Ciro como de las órdenes espartanas.

\section{A modo de conclusión}

Es imposible determinar las verdaderas causas que motivaron a miles de hombres a enrolarse en el primer gran ejército mercenario de la historia. No obstante la generalización socioeconómica queda estrecha en cuanto visión explicativa por los factores expuestos. En el presente trabajo se ha expuesto como las nuevas relaciones geográficas y geopolíticas, así como las relaciones de amistad

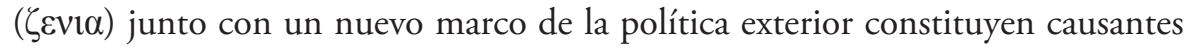
de dicho fenómeno histórico poco exploradas al día de hoy.

Por una parte, las relaciones geográficas que se dieron en el espacio geopolítico de la Hélade a inicios del siglo IV obligaron a que todos los actores políticos involucrados buscaran nuevas estrategias para acrecentar su poder. El espacio geopolítico, con sus hegemonías y por tanto formas de ejercer el poder espacialmente, fueron reflejo de estos mecanismos, los espartanos ante la escasez numérica militar de controlar un Imperio y ante el poder que poseían en el Peloponeso asumen la obligación de contratar mercenarios que sirvierontanto para cumplir pactos como para satisfacer la demanda de una política exterior activa.

Estas relaciones de poder de tipo geográfico tuvieron su reflejo en la $\zeta \varepsilon v i \alpha$. Más allá de la riqueza y de las condiciones socioeconómicas, los hombres que dispusieron la expedición de los diez mil tienen como denominador común que se encuentran allí por las relaciones de amistad y hospitalidad. Jenofonte, el autor de la principal fuente conservada de dicha expedición está por ejemplo en la expedición por lazos de hospitalidad que lo obligaron a enrolarse, sin ni siquiera conocer al líder rebelde. Esto demuestra cuán importante eran los lazos de sociabilidad en la Grecia antigua y como estos eran capaces de llegar a

${ }^{75}$ X. An7.2.2.

${ }^{76}$ X. An7.1.32. 
enrolar ejércitos completos. Bajo este prismaל̌vı $\alpha$ y riqueza no son fenómenos excluyentes, sino que fueron dos caras de una misma moneda puesto que los lazos de hospitalidad se generaron con vistas a ser usados y explotados para conseguir honor y bienestar material.

Más allá de la economía y la sociedad, los diez mil son reflejo de un mundo que había cambiado, como nos señalara Heródoto "los sucesos de los estados grandes y pequeños, visto que muchos, que antiguamente fueron grandes, han venido después a ser bien pequeños, y que, al contrario, fueron antes pequeños los que se han elevado en nuestros días a la mayor grandeza"77. Esta mutabilidad constante del mundo griego había trastocado las relaciones exteriores de las poleis, orientándolas a buscar un dominio territorial mucho más amplio del que estaban acostumbradas hacia fines de la Guerra del Peloponeso. La idea de un Imperio Espartano triunfante, que dominara la tierra antes que el mar se tradujo en la necesidad de contar con tropas activas para dicho dominio geográfico. Ciro y sus intenciones se avinieron a dicha política exterior, pues un conflicto interno dentro del Imperio Persa favorecía a los griegos, de la misma manera que la guerra del Peloponeso benefició a los persas y en particular a Ciro; la expedición de Agesilao demuestra la afirmación anterior.

La amistad, las relaciones sociales y un gran cambio geopolítico llevó a la constitución de los diez mil, cuestiones que aunadas por los deseos de Esparta de llevar a cabo una política expansiva y territorial en Jonia abrieron las puertas a un nuevo mecanismo para ejecutar la política exterior: el mercenarismo a gran escala.

\section{Referencias bibliográficas}

Austin M y Vidal-Naquet P. (1986) Economía y sociedad en la antigua Grecia. Barcelona: Paidos.

Baron C. (2006) "The Aristoteles Decree and the Expansion of the Second Athenian League". Hesperia: The Journal of the American School of Classical Studies at Athens, 75, 379-395.

Binttliff J. (1997) "Regional Survey, Demography, and the Rise of Complex

Societies in the Ancient Aegean: Core-Periphery, Neo-Malthusian, and Other Interpretive Models" Journal of Field Archaeology, 24, 1-38.

\footnotetext{
${ }^{77}$ Hdt 1.5 .
} 
Diodoro Sículo (2001-2012), Biblioteca Histórica, Madrid: Editorial Gredos.

Duran Badell M. (1998) "El mercenariado en la Grecia antigua”, Militaria. Revista de cultura militar, 12, 89-101, pp.90-91.

Estrabón, (1991-2003) Geografía. Madrid: Editorial Gredos.

Forni C. y Plácido D. (2009) “De la guerra del Peloponeso a la paz del Rey (III): los factores económicos públicos y privados en Atenas".Gerión, 27, 147-160.

Gómez Castro D. (2010) "El mercenario en el mundo griego a la luz de los estudios contemporáneos: reflexión teórica y nuevas tesis" Habis, 41,95-115.

Gómez Castro D. (2011) "La campaña egipcio-chipriota (383-373 a.c.): relaciones internacionales y mercenarios griegos en oriente". Gladius, 31, 4356.

Hanson V. "Epaminondas el tebano y la doctrina de la guerra preventiva" en Hanson V, ed. (2012) El arte de la guerra en el mundo antiguo: de las guerras persas a la caida de Roma. Barcelona: Crítica.

Heródoto de Halicarnaso, (2002) Los nueve libros de la historia. México: Porrua.

Hindley C. (1994) "Eros and Military Command in Xenophon" The Classical Quarterly, New Series, 44, 347-366.

JARDÉ. A (1960). La formación del pueblo griego, México: UTEHA. p. 116-117.

Jenofonte (1994) Las Helénicas. Madrid: Gredos.

Jenofonte (2001). Anábasis. Madrid: Gredos.

Justino, (1995) Epitome de las "historias filipicas" de Pompeyo Trogo/ Prólogos/ Fragmentos. Madrid: Editorial Gredos.

Kagan, D. (2003) Sobre las causas de la guerra y la preservación de la paz. México: FCE.

MARINOVIC L. (1998) Le mercenariatgrecau 4e siècle avantnotreère et la crise de la polis. Paris: Belles Lettres.

Miller H. (1984) "The Practical and Economic Background to the Greek Mercenary Explosion" Greece \& Rome, Second Series,31, 153-160.

Nussbaum G.B. (1967) The Ten Thousand: A Study in Social Organization and Action in Xenophon's Anabasis. Leiden, Holanda: EJ Bill.

PARke H.W. (1930) "The Development of the second spartan Empire (405-371 BC)", The Journal of Hellenic Studies, 50/1, 37-79. 
Plutarco, (1921) Vidas Paralelas, Traducción del Griego por Antonio Ranz Romanillos.

Radin M. (1911) "Xenophon's Ten Thousand" The Classical Journal, 7, 51-60.

Rouzé F y Amouretti M. (1992) El mundo griego antiguo, España: Akal.

Rusell A.G. (1942) “The Greek as a Mercenary Soldier” Greec \& Rome, 11/33, 103-112.

SAGE M. (2003). Warfare in ancient Greece: a sourcebook. Londres: Routledge.

Thompson. J. (1990) "State Practice, International Norms, and the Decline of Mercenarism”, International Studies Quarterly 34, 23-47,

Trundle M (2004). Greek Mercenaries from the Late Archai Period to Alexander. Inglaterra: Routledge.

Tucídides (1990/1992). Historia de la Guerra del Peloponeso. Obra completa. Madrid, Gredos.

Westlake H.D, (1936) “The Medism of Thessaly", The Journal of Hellenic Studies, 53, 12-24.

Whitehead D. (1991) “Who Equipped Mercenary Troops in Classical Greece?” Historia: Zeitschriftfür Alte Geschichte, 40,105-113. 